\title{
Identifying Liver Cancer-Related Enhancer SNPs by Integrating GWAS and Histone Modification ChIP-seq Data
}

\author{
Tianjiao Zhang, ${ }^{1}$ Yang $\mathrm{Hu}^{2}{ }^{2}$ Xiaoliang Wu, ${ }^{1}$ Rui Ma, ${ }^{1}$ Qinghua Jiang, ${ }^{2}$ and Yadong Wang ${ }^{1}$ \\ ${ }^{1}$ School of Computer Science and Technology, Harbin Institute of Technology, Harbin 150001, China \\ ${ }^{2}$ School of Life Science and Technology, Harbin Institute of Technology, Harbin 150001, China \\ Correspondence should be addressed to Qinghua Jiang; qhjiang@hit.edu.cn and Yadong Wang; ydwang@hit.edu.cn
}

Received 9 April 2016; Revised 30 May 2016; Accepted 1 June 2016

Academic Editor: Xun Lan

Copyright (c) 2016 Tianjiao Zhang et al. This is an open access article distributed under the Creative Commons Attribution License, which permits unrestricted use, distribution, and reproduction in any medium, provided the original work is properly cited.

\begin{abstract}
Many disease-related single nucleotide polymorphisms (SNPs) have been inferred from genome-wide association studies (GWAS) in recent years. Numerous studies have shown that some SNPs located in protein-coding regions are associated with numerous diseases by affecting gene expression. However, in noncoding regions, the mechanism of how SNPs contribute to disease susceptibility remains unclear. Enhancer elements are functional segments of DNA located in noncoding regions that play an important role in regulating gene expression. The SNPs located in enhancer elements may affect gene expression and lead to disease. We presented a method for identifying liver cancer-related enhancer SNPs through integrating GWAS and histone modification ChIP-seq data. We identified 22 liver cancer-related enhancer SNPs, 9 of which were regulatory SNPs involved in distal transcriptional regulation. The results highlight that these enhancer SNPs may play important roles in liver cancer.
\end{abstract}

\section{Introduction}

Single nucleotide polymorphism (SNP) is a variation at a single nucleotide in a DNA sequence [1]. In the last decade, a large number of genome-wide association studies (GWAS) have been published, indicating that thousands of SNPs are associated with diseases. Linkage disequilibrium is the nonrandom association of alleles at different genome locations [2]. There are many SNPs in LD with the causal SNP at specific GWAS locus [3, 4]. Over $90 \%$ of these GWAS variants are located in noncoding regions, and approximately $10 \%$ are in $\mathrm{LD}$ with a protein-coding variant $[5,6]$. In protein-coding regions, many studies have shown that some SNPs are associated with numerous diseases by affecting gene expression $[7,8]$. However, in noncoding regions, the mechanism of how SNPs contribute to disease susceptibility remains unclear.

Enhancers are the core regulatory components of the genome that act over a distance to positively regulate gene expression [9]. It is estimated that 400,000 to 1 million putative enhancers exist in the human genome [10,11]. Recently, some studies have shown that disease-related GWAS SNPs are correlated with enhancers marked with special histone modifications [12-15]. Therefore, through integrating GWAS and histone modification ChIP-seq data in a given disorder, we can identify disease-related enhancer SNPs.

We provided a method for identifying liver cancer-related enhancer SNPs through integrating liver cancer GWAS and histone modification ChIP-seq data. We identified 22 enhancer SNPs associated with liver cancer, 9 of which were regulatory SNPs involved in distal transcriptional regulation. The results highlight that these enhancer SNPs may play important roles in liver cancer.

\section{Results}

2.1. Pipeline of Identifying Liver Cancer-Related Enhancer SNPs. As shown in Figure 1, the pipeline consists of four steps. Firstly, we downloaded liver cancer-related GWAS SNPs from the GRASP [16] database and used LD data from HapMap [17] to infer liver cancer-related LD SNPs. Secondly, we identified enhancer regions in liver cancer through integrating histone modification ChIP-seq data in 


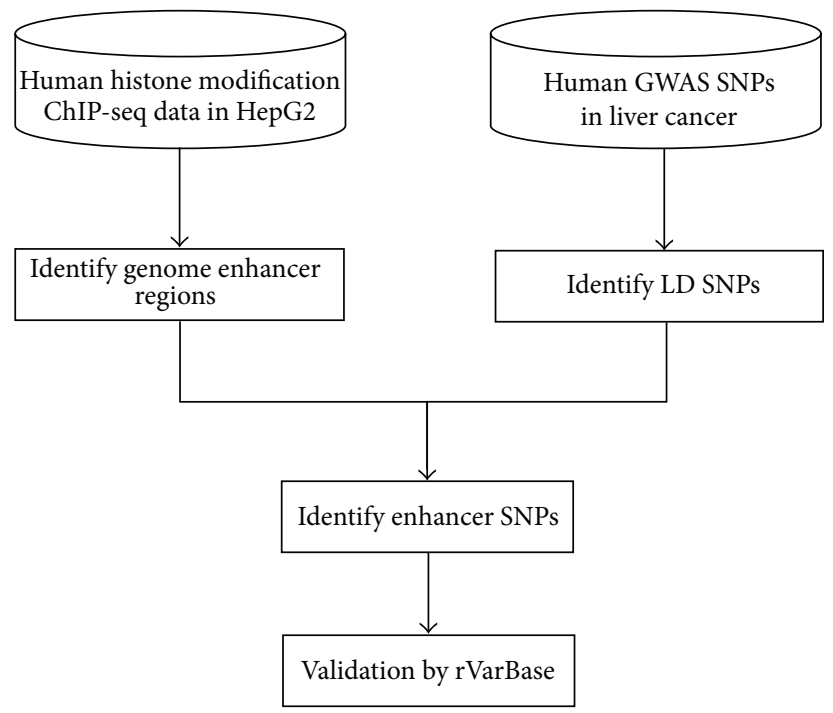

FIGURE 1: Flowchart identifying liver cancer-related enhancer SNPs. LD SNPs: SNPs located in linkage disequilibrium blocks of GWAS SNPs. Enhancer SNPs: SNPs identified in enhancer regions. rVarBase: a database for variant's regulatory features.

the HepG2 cell line. Thirdly, we mapped the liver cancerrelated LD SNPs to the identified enhancers in liver cancer and obtained liver cancer-related enhancer SNPs. Finally, we used a curated regulatory SNP database named rVarBase [18] to validate our results.

2.2. Linkage Disequilibrium Analysis with Liver CancerAssociated SNPs. We obtained 45 liver cancer-associated SNPs from GRASP (Table 1). These SNPs are the raw potential liver cancer-related SNPs. Then, we used LD data from HapMap to achieve liver cancer-associated LD SNPs. The total number of potential liver cancer-related SNPs is 340 .

2.3. Identification of Liver Cancer-Related Enhancer SNPs. Previous studies indicated that the enhancer regions are marked by a strong $\mathrm{H} 3 \mathrm{~K} 4 \mathrm{mel}$ signal and a relatively weak H3K4me3 signal $[19,20]$. Thus, we used histone modification ChIP-seq data to recognize the enhancer regions in liver cancer. Then, we mapped the liver cancer-related GWAS SNPs to the enhancer regions and obtained 22 enhancer SNPs in liver cancer (Table 2).

2.4. Validation as Regulatory SNPs. rVarBase is a database that provides reliable, comprehensive, and user-friendly annotations on variant's regulatory features [18]. It includes regulatory SNPs (rSNPs), LD-proxies of rSNPs, and genes that are potentially regulated by rSNPs. We used rVarBase to analyze these 22 enhancer SNPs in liver cancer and found that 14 SNPs have evidence of regulatory SNPs and 9 SNPs (rs9494257, rs6903949, rs6996881, rs4739519, rs6988263, rs12156293, rs1568658, rs5994449, and rs5753816) are involved in distal transcriptional regulation (Table 3). Table 4 shows the potential target genes of these 9 SNPs.

\section{Materials and Methods}

3.1. GWAS and LD Datasets. We downloaded the human liver cancer-related GWAS SNPs from GRASP. The database includes 26 and 19 liver cancer-associated SNPs $(p<$ $10^{-5}$ ) from Han Chinese in Beijing, China (CHB), and Japanese in Tokyo, Japan (JPT), respectively. The URL is https:/grasp.nhlbi.nih.gov/Overview.aspx. We obtained all SNPs in LD with GWAS-lead SNPs using LD blocks identified with publicly available HapMap data on the CHB and JPT populations. The LD data can be downloaded from http://hapmap.ncbi.nlm.nih.gov/index.html/.

3.2. Histone Modification Datasets. We downloaded the human histone modification ChIP-seq datasets in the HepG2 cell line from the ENCODE Production Data/ Broad Institute. The URL is http://genome.ucsc.edu/ ENCODE/downloads.html.

3.3. Linkage Disequilibrium Analysis. In the genome, SNPs located in close proximity tend to be in linkage disequilibrium with each other. The International HapMap Project has established linkage disequilibrium of human genome SNPs. We used LD data from HapMap to achieve liver cancerassociated LD SNPs $\left(R^{2}>0.8\right)$.

3.4. Identify Enhancer Regions and Enhancer SNPs. Firstly, we downloaded the human histone modification BAM files (H3K4mel and H3K4me3) in the HepG2 cell line from the ENCODE project. Then, we used BEDtools [21] to count read coverage for every position of the genome. Through calculating the ratio $\mathrm{H} 3 \mathrm{~K} 4 \mathrm{mel} / \mathrm{H} 3 \mathrm{~K} 4 \mathrm{me} 3$ and picking up the regions with $\log _{2}(\mathrm{H} 3 \mathrm{~K} 4 \mathrm{me} / \mathrm{H} 3 \mathrm{~K} 4 \mathrm{me} 3)>1.2$, we identified the potential enhancer regions. Finally, we mapped the potential LD SNPs to these enhancer regions and achieved liver cancer-related enhancer SNPs. 
TABLE 1: Summary of liver cancer-associated SNPs from GRASP database.

\begin{tabular}{|c|c|c|c|c|}
\hline SNP ID & $p$ value & Chromosome & Populations & PMID \\
\hline rs17401966 & $1.20 E-19$ & 1 & $\mathrm{CHB}$ & 20676096 \\
\hline rs1249458 & $8.30 E-06$ & 2 & $\mathrm{CHB}$ & 22807686 \\
\hline rs1714259 & $1.10 E-06$ & 2 & $\mathrm{CHB}$ & 22807686 \\
\hline rs2396470 & $5.10 E-07$ & 2 & $\mathrm{CHB}$ & 20676096 \\
\hline rs7424161 & $8.80 E-06$ & 2 & $\mathrm{CHB}$ & 22807686 \\
\hline rs7574865 & $1.70 E-11$ & 2 & $\mathrm{CHB}$ & 23242368 \\
\hline rs3905886 & $3.70 E-06$ & 3 & $\mathrm{CHB}$ & 22807686 \\
\hline rs1073547 & $6.80 E-06$ & 4 & $\mathrm{CHB}$ & 22807686 \\
\hline rs17081345 & $3.70 E-07$ & 6 & $\mathrm{CHB}$ & 20676096 \\
\hline rs9272105 & $3.30 E-23$ & 6 & $\mathrm{CHB}$ & 22807686 \\
\hline rs9275319 & $8.70 E-19$ & 6 & $\mathrm{CHB}$ & 23242368 \\
\hline rs9494257 & $1.10 E-14$ & 6 & $\mathrm{CHB}$ & 20676096 \\
\hline rs12682266 & $6.70 E-06$ & 8 & $\mathrm{CHB}$ & 22174901 \\
\hline rs1573266 & $7.40 E-06$ & 8 & $\mathrm{CHB}$ & 22174901 \\
\hline rs2275959 & $6.40 E-06$ & 8 & $\mathrm{CHB}$ & 22174901 \\
\hline rs7821974 & $7.00 E-06$ & 8 & $\mathrm{CHB}$ & 22174901 \\
\hline rs7898005 & $7.00 E-08$ & 10 & $\mathrm{CHB}$ & 20676096 \\
\hline rs10160758 & $6.00 E-06$ & 11 & $\mathrm{CHB}$ & 22807686 \\
\hline rs10896464 & $6.50 E-06$ & 11 & $\mathrm{CHB}$ & 22807686 \\
\hline rs2611145 & $9.30 E-06$ & 11 & $\mathrm{CHB}$ & 22174901 \\
\hline rs3825023 & $3.10 E-06$ & 11 & $\mathrm{CHB}$ & 22807686 \\
\hline rs7119426 & $3.50 E-06$ & 11 & $\mathrm{CHB}$ & 22807686 \\
\hline rs402071 & $8.60 E-06$ & 19 & $\mathrm{CHB}$ & 22807686 \\
\hline rs3092194 & $4.40 E-06$ & 20 & $\mathrm{CHB}$ & 22807686 \\
\hline rs368007 & $9.90 E-06$ & 20 & $\mathrm{CHB}$ & 22807686 \\
\hline rs 455804 & $4.40 E-10$ & 21 & $\mathrm{CHB}$ & 22807686 \\
\hline rs1980215 & $2.30 E-06$ & 3 & JPT & 21499248 \\
\hline rs 2596542 & $4.20 E-13$ & 6 & JPT & 21499248 \\
\hline rs9275572 & $1.40 E-09$ & 6 & JPT & 21499248 \\
\hline rs1568658 & $6.90 E-06$ & 7 & JPT & 21499248 \\
\hline rs952656 & $2.80 E-06$ & 8 & JPT & 21499248 \\
\hline rs4363614 & $4.20 E-07$ & 11 & JPT & 21499248 \\
\hline rs1957496 & $4.60 E-06$ & 14 & JPT & 21499248 \\
\hline rs8019534 & $3.90 E-06$ & 14 & JPT & 21499248 \\
\hline rs1794304 & $3.60 E-06$ & 16 & JPT & 21725309 \\
\hline rs2208456 & $3.80 E-06$ & 20 & JPT & 21499248 \\
\hline rs1012068 & $1.30 E-14$ & 22 & JPT & 21725309 \\
\hline rs11703779 & $4.20 E-06$ & 22 & JPT & 21725309 \\
\hline rs4820994 & $3.00 E-06$ & 22 & JPT & 21725309 \\
\hline rs4820996 & $4.20 E-06$ & 22 & JPT & 21725309 \\
\hline rs5753816 & $4.90 E-06$ & 22 & JPT & 21725309 \\
\hline rs5753818 & $9.40 E-06$ & 22 & JPT & 21725309 \\
\hline rs5998152 & $1.20 E-07$ & 22 & JPT & 21725309 \\
\hline rs7287054 & $3.80 E-06$ & 22 & JPT & 21725309 \\
\hline rs737084 & $5.90 E-06$ & 22 & JPT & 21725309 \\
\hline
\end{tabular}

\section{Discussion}

Through integrating liver cancer GWAS SNPs from GRASP, LD data from HapMap, and histone modification ChIP-seq data from ENCODE, we explored liver cancer-related enhancer SNPs. We compared our results with rVarBase and found that 9 SNPs (rs9494257, rs6903949, rs6996881, rs4739519, rs6988263, rs12156293, rs1568658, rs5994449, 
TABLE 2: Summary of predicted enhancer SNPs in liver cancer.

\begin{tabular}{|c|c|c|c|c|c|}
\hline SNP ID & Chromosome & Start & End & Chain & Populations \\
\hline rs12751375 & chrl & 10291873 & 10291874 & + & $\mathrm{CHB}$ \\
\hline rs6700866 & chrl & 10306037 & 10306038 & + & $\mathrm{CHB}$ \\
\hline rs9494257 & chr6 & 135827471 & 135827472 & + & $\mathrm{CHB}$ \\
\hline rs17064474 & chr6 & 135680137 & 135680138 & + & $\mathrm{CHB}$ \\
\hline rs17721919 & chr6 & 135748923 & 135748924 & + & $\mathrm{CHB}$ \\
\hline rs17721931 & chr6 & 135749377 & 135749378 & + & $\mathrm{CHB}$ \\
\hline rs6903949 & chr6 & 135821065 & 135821066 & + & $\mathrm{CHB}$ \\
\hline rs6996881 & chr8 & 37407919 & 37407920 & + & $\mathrm{CHB}$ \\
\hline rs4739519 & chr8 & 37412858 & 37412859 & + & $\mathrm{CHB}$ \\
\hline rs6988263 & chr8 & 37414659 & 37414660 & + & $\mathrm{CHB}$ \\
\hline rs12156293 & chr8 & 37419921 & 37419922 & + & $\mathrm{CHB}$ \\
\hline rs6928810 & chr6 & 31410523 & 31410524 & + & JPT \\
\hline rs3869132 & chr6 & 31410947 & 31410948 & - & JPT \\
\hline rs2596562 & chr6 & 31354594 & 31354595 & - & JPT \\
\hline rs2523475 & chr6 & 31361709 & 31361710 & - & JPT \\
\hline rs2523467 & chr6 & 31362929 & 31362930 & - & $\mathrm{JPT}$ \\
\hline rs9501387 & chr6 & 31364458 & 31364459 & + & JPT \\
\hline rs1568658 & chr7 & 29141557 & 29141558 & - & JPT \\
\hline rs1794304 & chr16 & 12625394 & 12625395 & + & JPT \\
\hline rs5994449 & $\operatorname{chr} 22$ & 32304178 & 32304179 & + & JPT \\
\hline rs5753816 & $\operatorname{chr} 22$ & 32312841 & 32312842 & + & JPT \\
\hline rs5749339 & chr22 & 32315734 & 32315735 & + & JPT \\
\hline
\end{tabular}

TABLE 3: Summary of liver cancer-related regulatory SNPs validated by rVarBase.

\begin{tabular}{|c|c|c|c|c|}
\hline SNP ID & Regulatory SNP & Distal regulation & Chromatin state & Related regulatory elements \\
\hline rs12751375 & Yes & No & Inactive region & $\mathrm{n} / \mathrm{a}$ \\
\hline rs6700866 & Yes & No & $\begin{array}{l}\text { Weak transcription; ZNF genes and } \\
\text { repeats; strong transcription; } \\
\text { enhancers }\end{array}$ & $\mathrm{n} / \mathrm{a}$ \\
\hline rs9494257 & Yes & Yes & $\begin{array}{c}\text { Enhancers; flanking active TSS; } \\
\text { weak transcription }\end{array}$ & Chromatin interactive region \\
\hline rs17064474 & Yes & No & $\begin{array}{l}\text { Weak transcription; active TSS; } \\
\text { flanking active TSS; enhancers }\end{array}$ & $\mathrm{n} / \mathrm{a}$ \\
\hline rs17721919 & Yes & No & Weak transcription & $\mathrm{n} / \mathrm{a}$ \\
\hline rs17721931 & Yes & No & Weak transcription & $\mathrm{n} / \mathrm{a}$ \\
\hline rs6903949 & Yes & Yes & Weak transcription; enhancers & TF binding region; chromatin interactive region \\
\hline rs6996881 & Yes & Yes & Weak transcription; enhancers & Chromatin interactive region \\
\hline rs4739519 & Yes & Yes & Enhancers; weak transcription & Chromatin interactive region \\
\hline rs6988263 & Yes & Yes & $\begin{array}{c}\text { Enhancers; weak transcription; } \\
\text { genic enhancers; bivalent enhancer; } \\
\text { flanking active TSS }\end{array}$ & Chromatin interactive region \\
\hline rs12156293 & Yes & Yes & $\begin{array}{l}\text { Enhancers; weak transcription; } \\
\text { bivalent enhancer; genic enhancers }\end{array}$ & Chromatin interactive region \\
\hline rs1568658 & Yes & Yes & $\begin{array}{l}\text { Weak transcription; enhancers; } \\
\text { strong transcription }\end{array}$ & Chromatin interactive region \\
\hline rs5994449 & Yes & Yes & $\begin{array}{c}\text { Weak transcription; strong } \\
\text { transcription; ZNF genes and } \\
\text { repeats }\end{array}$ & Chromatin interactive region \\
\hline rs5753816 & Yes & Yes & $\begin{array}{l}\text { Weak transcription; enhancers; } \\
\text { flanking active TSS }\end{array}$ & Chromatin interactive region \\
\hline
\end{tabular}


TABLE 4: Summary of liver cancer-related regulatory SNPs and potential target genes validated by rVarBase.

\begin{tabular}{|c|c|c|c|}
\hline SNP ID & Gene symbol & Ensemble ID & Regulation type \\
\hline rs9494257 & BCLAF1 & ENSG00000029363 & Distal transcriptional regulation \\
\hline rs9494257 & AHI1 & ENSG00000135541 & Distal transcriptional regulation \\
\hline rs9494257 & LINC00271 & ENSG00000231028 & Distal transcriptional regulation \\
\hline rs6903949 & MYB & ENSG00000118513 & Distal transcriptional regulation \\
\hline rs6903949 & BCLAF1 & ENSG00000029363 & Distal transcriptional regulation \\
\hline rs6903949 & AHI1 & ENSG00000135541 & Distal transcriptional regulation \\
\hline rs6903949 & LINC00271 & ENSG00000231028 & Distal transcriptional regulation \\
\hline rs6996881 & ZNF703 & ENSG00000183779 & Distal transcriptional regulation \\
\hline rs6996881 & ERLIN2 & ENSG00000147475 & Distal transcriptional regulation \\
\hline rs6996881 & Null & ENSG00000183154 & Distal transcriptional regulation \\
\hline rs6996881 & Null & ENSG00000253161 & Distal transcriptional regulation \\
\hline rs4739519 & ZNF703 & ENSG00000183779 & Distal transcriptional regulation \\
\hline rs4739519 & Null & ENSG00000254290 & Distal transcriptional regulation \\
\hline rs6988263 & ZNF703 & ENSG00000183779 & Distal transcriptional regulation \\
\hline rs6988263 & Null & ENSG00000254290 & Distal transcriptional regulation \\
\hline rs12156293 & ZNF703 & ENSG00000183779 & Distal transcriptional regulation \\
\hline rs12156293 & Null & ENSG00000254290 & Distal transcriptional regulation \\
\hline rs12156293 & ERLIN2 & ENSG00000147475 & Distal transcriptional regulation \\
\hline rs12156293 & Null & ENSG00000183154 & Distal transcriptional regulation \\
\hline rs1568658 & Null & ENSG00000228421 & Distal transcriptional regulation \\
\hline rs1568658 & TRIL & ENSG00000176734 & Distal transcriptional regulation \\
\hline rs1568658 & Null & ENSG00000255690 & Distal transcriptional regulation \\
\hline rs5994449 & DEPDC5 & ENSG00000100150 & Distal transcriptional regulation \\
\hline rs5994449 & FBXO7 & ENSG00000100225 & Distal transcriptional regulation \\
\hline rs5994449 & SYN3 & ENSG00000185666 & Distal transcriptional regulation \\
\hline rs5994449 & PRR14L & ENSG00000183530 & Distal transcriptional regulation \\
\hline rs5994449 & PISD & ENSG00000241878 & Distal transcriptional regulation \\
\hline rs5994449 & EIF4ENIF1 & ENSG00000184708 & Distal transcriptional regulation \\
\hline rs5994449 & RNU6-28 & ENSG00000199248 & Distal transcriptional regulation \\
\hline rs5994449 & SFI1 & ENSG00000198089 & Distal transcriptional regulation \\
\hline rs5753816 & YWHAH & ENSG00000128245 & Distal transcriptional regulation \\
\hline rs5753816 & C22orf24 & ENSG00000128254 & Distal transcriptional regulation \\
\hline rs5753816 & PISD & ENSG00000241878 & Distal transcriptional regulation \\
\hline rs5753816 & DEPDC5 & ENSG00000100150 & Distal transcriptional regulation \\
\hline rs5753816 & RNU6-28 & ENSG00000199248 & Distal transcriptional regulation \\
\hline rs5753816 & SFI1 & ENSG00000198089 & Distal transcriptional regulation \\
\hline rs5753816 & EIF4ENIF1 & ENSG00000184708 & Distal transcriptional regulation \\
\hline rs5753816 & RFPL3S & ENSG00000205853 & Distal transcriptional regulation \\
\hline rs5753816 & Null & ENSG00000230736 & Distal transcriptional regulation \\
\hline rs5753816 & Null & ENSG00000243519 & Distal transcriptional regulation \\
\hline rs5753816 & Null & ENSG00000241954 & Distal transcriptional regulation \\
\hline rs5753816 & Null & ENSG00000232218 & Distal transcriptional regulation \\
\hline rs5753816 & SYN3 & ENSG00000185666 & Distal transcriptional regulation \\
\hline
\end{tabular}

and rs5753816) were regulatory SNPs involved in distal transcriptional regulation. The results highlight that these enhancer SNPs may play important roles in liver cancer.

Compared with protein-coding regions in the human genome, noncoding regions contain much more genetic variations. Some important regulation regions, such as enhancers, have great influence on target gene expression.
SNPs located in these regions may disturb gene expression and even cause diseases. Thus, the identification of SNPs in enhancer regions is helpful to understand the mechanism of association between SNPs and diseases.

We presented a method to identify disease-related SNPs located in enhancer regions that gives a new solution to investigate the relationship between SNPs and diseases. The 
presented method can also be applied to other diseases and will enable biologists to investigate the mechanism of disease risk associated with SNPs.

\section{Competing Interests}

The authors declare that there are no competing interests regarding the publication of this paper.

\section{Authors' Contributions}

Tianjiao Zhang collected the data, designed the computational experiments, carried out the statistical analysis, and wrote the paper. Qinghua Jiang participated in the design of the study. Yang Hu, Xiaoliang Wu, and Rui Ma participated in the revision of this paper. Yadong Wang gave comments and revisions to the final version of this paper. All authors read and approved the final paper. Tianjiao Zhang and Yang Hu equally contributed to this paper.

\section{Acknowledgments}

This work was partially supported by the National High-Tech Research and Development Program (863) of China (2012AA02A601, 2012AA02A602, 2012AA020404, 2012AA020409, 2012AA02A604, 2014AA021505, 2015AA020101, and 2015AA020108), National Science and Technology Major Project [no. 2013ZX03005012], and the National Natural Science Foundation of China (61571152, 31301089).

\section{References}

[1] R. Sachidanandam, D. Weissman, S. C. Schmidt et al., "A map of human genome sequence variation containing 1.42 million single nucleotide polymorphisms," Nature, vol. 409, no. 6822, pp. 928-933, 2001.

[2] M. Slatkin, "Linkage disequilibrium-understanding the evolutionary past and mapping the medical future," Nature Reviews Genetics, vol. 9, no. 6, pp. 477-485, 2008.

[3] J. Peng, S. Uygun, T. Kim, Y. Wang, S. Y. Rhee, and J. Chen, "Measuring semantic similarities by combining gene ontology annotations and gene co-function networks," BMC Bioinformatics, vol. 16, no. 1, article 44, 2015.

[4] J. Peng, T. Wang, J. Wang, Y. Wang, and J. Chen, "Extending gene ontology with gene association networks," Bioinformatics, vol. 32, no. 8, pp. 1185-1194, 2016.

[5] M. T. Maurano, R. Humbert, E. Rynes et al., "Systematic localization of common disease-associated variation in regulatory DNA," Science, vol. 337, no. 6099, pp. 1190-1195, 2012.

[6] M. A. Schaub, A. P. Boyle, A. Kundaje, S. Batzoglou, and M. Snyder, "Linking disease associations with regulatory information in the human genome," Genome Research, vol. 22, no. 9, pp. 1748-1759, 2012.

[7] L. A. Hindorff, P. Sethupathy, H. A. Junkins et al., "Potential etiologic and functional implications of genome-wide association loci for human diseases and traits," Proceedings of the National Academy of Sciences of the United States of America, vol. 106, no. 23, pp. 9362-9367, 2009.
[8] Q. Zou, J. Li, Q. Hong et al., "Prediction of microRNA-disease associations based on social network analysis methods," BioMed Research International, vol. 2015, Article ID 810514, 9 pages, 2015.

[9] J. Banerji, S. Rusconi, and W. Schaffner, "Expression of a $\beta$ globin gene is enhanced by remote SV40 DNA sequences," Cell, vol. 27, no. 2, pp. 299-308, 1981.

[10] C. Buecker and J. Wysocka, "Enhancers as information integration hubs in development: lessons from genomics," Trends in Genetics, vol. 28, no. 6, pp. 276-284, 2012.

[11] W. Xie and B. Ren, "Developmental biology. Enhancing pluripotency and lineage specification," Science, vol. 341, no. 6143, pp. 245-247, 2013.

[12] S. Heinz, C. Benner, N. Spann et al., "Simple combinations of lineage-determining transcription factors prime cis-regulatory elements required for macrophage and B cell identities," Molecular Cell, vol. 38, no. 4, pp. 576-589, 2010.

[13] J. Ernst, P. Kheradpour, T. S. Mikkelsen et al., "Mapping and analysis of chromatin state dynamics in nine human cell types," Nature, vol. 473, no. 7345, pp. 43-49, 2011.

[14] B. Akhtar-Zaidi, R. Cowper-Sallari, O. Corradin et al., "Epigenomic enhancer profiling defines a signature of colon cancer," Science, vol. 336, no. 6082, pp. 736-739, 2012.

[15] G. Trynka, C. Sandor, B. Han et al., "Chromatin marks identify critical cell types for fine mapping complex trait variants," Nature Genetics, vol. 45, no. 2, pp. 124-130, 2013.

[16] R. Leslie, C. J. O’Donnell, and A. D. Johnson, “GRASP: analysis of genotype-phenotype results from 1390 genome-wide association studies and corresponding open access database," Bioinformatics, vol. 30, no. 12, pp. i185-i194, 2014.

[17] The International HapMap Consortium, "The international HapMap project," Nature, vol. 426, no. 6968, pp. 789-796, 2003.

[18] L. Guo, Y. Du, S. Qu, and J. Wang, "rVarBase: an updated database for regulatory features of human variants," Nucleic Acids Research, vol. 44, no. 1, pp. D888-D893, 2016.

[19] F. de Santa, I. Barozzi, F. Mietton et al., "A large fraction of extragenic RNA Pol II transcription sites overlap enhancers," PLoS Biology, vol. 8, no. 5, article e1000384, 2010.

[20] A. C. Marques, J. Hughes, B. Graham, M. S. Kowalczyk, D. R. Higgs, and C. P. Ponting, "Chromatin signatures at transcriptional start sites separate two equally populated yet distinct classes of intergenic long noncoding RNAs," Genome Biology, vol. 14, no. 11, article R131, 2013.

[21] A. R. Quinlan, "UNIT 11.12 BEDTools: the swiss-army tool for genome feature analysis," Current Protocols in Bioinformatics, vol. 47, pp. 11.12.1-11.12.34, 2014. 

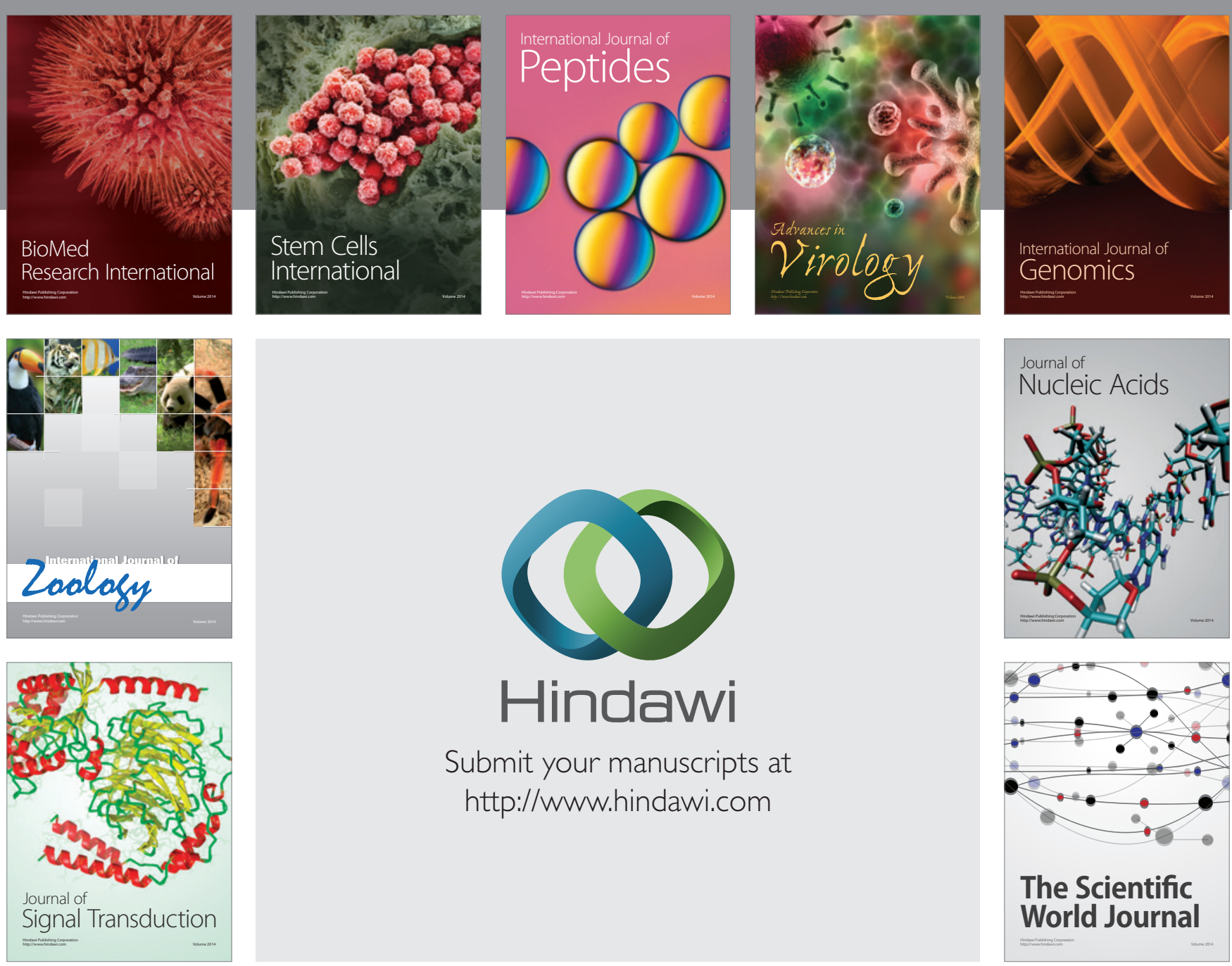

Submit your manuscripts at

http://www.hindawi.com
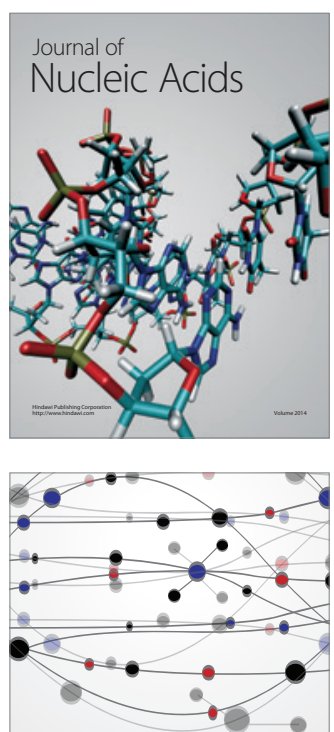

The Scientific World Journal
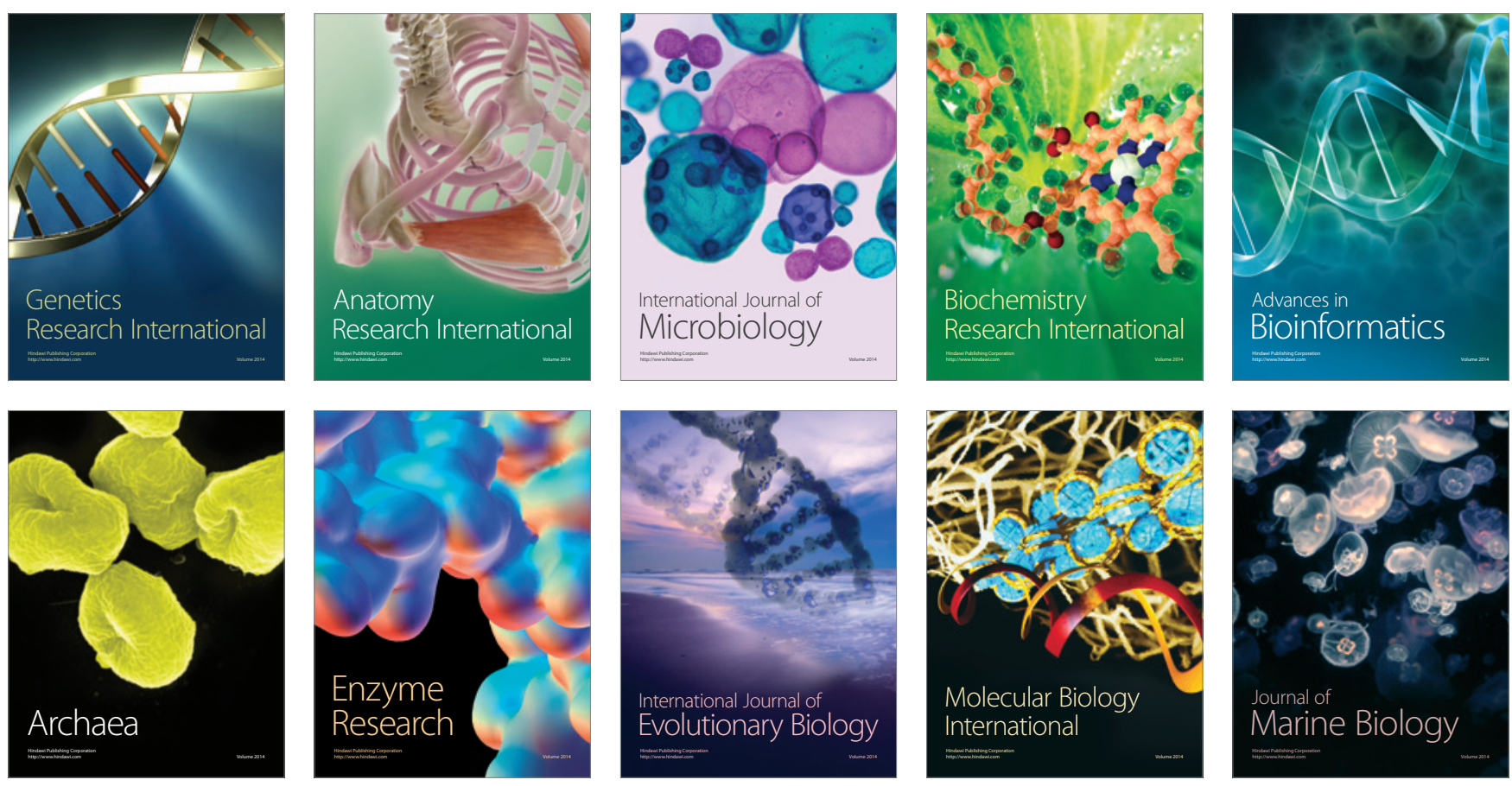